\title{
De l'anglais au français en langue de spécialité économique : équivalences attestées et détours des choix traductologiques
}

Jacqueline Percebois

\section{(2) OpenEdition}

\section{Journals}

Édition électronique

URL : http://journals.openedition.org/asp/1055

DOI : 10.4000/asp.1055

ISBN : 978-2-8218-0398-5

ISSN : 2108-6354

\section{Éditeur}

Groupe d'étude et de recherche en anglais de spécialité

\section{Édition imprimée}

Date de publication : 1 décembre 2004

Pagination : 81-95

ISSN : 1246-8185

Référence électronique

Jacqueline Percebois, « De l'anglais au français en langue de spécialité économique : équivalences attestées et détours des choix traductologiques », ASp [En ligne], 45-46 | 2004, mis en ligne le 28 mars 2010, consulté le 19 avril 2019. URL : http://journals.openedition.org/asp/1055 ; DOI : 10.4000/ asp. 1055

Ce document a été généré automatiquement le 19 avril 2019

Tous droits réservés 


\title{
De l'anglais au français en langue de spécialité économique : équivalences attestées et détours des choix traductologiques
}

\author{
Jacqueline Percebois
}

\section{Introduction}

1 Cet article traite de la traduction de textes anglais de spécialité économique et se propose d'aborder certains principes de traductologie. Néologisme qui désigne l'étude théorique de la traduction (Pergnier 1993: 7), la traductologie vise à « définir les principes et les règles régissant la reformulation d'un message d'une langue en une autre » (Delisle 1993 : 48). Dans une première partie, nous définirons langue de spécialité, traduction et traduction en langue de spécialité, en établissant une relation entre la notion de fidélité et les circonstances de la traduction. La deuxième partie exposera les étapes de la traduction en signalant les aspects spécifiques liés à la langue de spécialité économique. Enfin, dans la troisième partie de cet article, nous préciserons ce que nous entendons par « équivalences attestées » et donnerons des exemples des détours dont résultent les choix traductologiques.

\section{Traduire l'anglais de spécialité économique}

\subsection{Qu'est-ce que traduire?}

2 Les réponses des spécialistes à cette question font toutes apparaître les termes « communication », «médiation » (ou «médiateur ») et «message ». Nous citerons trois références en la matière par ordre chronologique. 
En 1993, Maurice Pergnier écrit que la traduction inclut « tous les faits de communication dans lesquels une information formulée dans un code est reformulée au moyen d'un code différent» (1993: 27), le traducteur étant, par définition, un "médiateur de la communication » (1993: 50). Nous retenons ici l'idée de la reformulation de l'information et du passage d'un code à un autre code.

En 1994, Jean-René Ladmiral écrit :

La traduction est un cas particulier de convergence linguistique : au sens le plus large, elle désigne toute forme de «médiation linguistique», permettant de transmettre de l'information entre locuteurs de langues différentes. La traduction fait passer un message d'une langue de départ (LD) ou langue-source dans une langue d'arrivée (LA) ou langue-cible. (1994:11)

Cette définition insiste sur la transmission de l'information, le passage du message d'une langue à une autre langue, d'un texte de départ (TD) à un texte d'arrivée (TA).

Enfin, Danica Seleskovitch et Marianne Lederer (2001) estiment que «traduire ce n'est pas seulement transformer des signes en d'autres signes", «il faut au préalable, déterminer la signification pertinente de ces signes pour trouver la correspondance dans l'autre langue ». Une fois levée la polysémie des mots et l'ambiguïté des phrases, il ne peut être question pour le traducteur de se lancer dans la rédaction du texte d'arrivée « tant que les signes linguistiques ne se combinent pas en un message » (2001:15).

7 En définitive, la reformulation de l'information d'un code à l'autre permet la transmission du message contenu dans le texte source qui s'effectue ainsi d'une langue à une autre langue. Cette transmission ne peut s'effectuer qu'une fois que, le sens des signes et des phrases ayant été décrypté, les éléments composant le texte d'arrivée réunis par le traducteur forment un message cohérent. Telles sont les notions définies pour la traduction non spécialisée.

\subsection{La langue de spécialité économique}

8 La langue de spécialité économiqueest un domaine particulier constitué de sousdomaines. Une analyse bottom-up permet d'observer qu'elle est constituée d'un lexique économique intégré dans une phraséologie typique pour chacun des sous-domaines qui la composent. Ainsi peut-on considérer le domaine économique divisé en deux parties et étudier les différents aspects de l'anglais de spécialité micro- et macro-économique, l'anglais financier, l'anglais des échanges commerciaux, des transports, de l'environnement, etc. Chacun de ces domaines constitue, d'une part, un sujet d'étude particulier sur le plan linguistique et, d'autre part, se compose d'éléments qui pourront constituer un sous-domaine linguistique d'autres grands domaines: par exemple, la terminologie financière est un thème d'étude à part entière mais se retrouve aussi comme élément constitutif de la plupart des grands domaines économiques; c'est alors la terminologie financière appliquée, par exemple, aux transports ou aux échanges internationaux.

\subsection{Qu'en est-il de la traduction en langue de spécialité ?}

9 Qu'en est-il de la traduction en langue de spécialité ?En règle générale, communication, médiation et message forment la trame de la traductologie et sont l'objet de tous les soins 
du traducteur. En langue de spécialité, s'y ajoute la connaissance des notions et dénominations propres au domaine d'application.

Pierre Lerat préfère le terme de « langue spécialisée » à celui de « langue de spécialité »; il parle ensuite naturellement de «traduction spécialisée ». Pour lui, dans ce domaine, la connaissance des notions est un pré-requis :

On comprend que la traduction spécialisée soit de plus en plus l'affaire de spécialistes du domaine plus ou moins bilingues. Pour la logique du texte, ce sont effectivement les meilleurs juges. De même, nul n'est mieux placé qu'eux pour connaître les dénominations usuelles dans la profession ou le milieu concernés. Le

« plus » du traducteur de métier ne peut donc être que linguistique. (1995 : 100-101)

En outre, P. Lerat considère que le traducteur dispose d'un triple avantage : la maîtrise des distributions au niveau du syntagme, l'habitude de la gymnastique rédactionnelle de la paraphrase et la familiarité avec le fonctionnement de la langue (1995 : 101-102). Ainsi le traducteur peut-il comparer les collocations et les diverses options de paraphrases déterminées par sa connaissance de la terminologie et de la syntaxe.

Le traducteur veillera à la cohérence de la terminologie sur le plan synchronique et diachronique. Pour le respect de la «cohérence synchronique», il doit associer dénominations et concepts dans chaque langue afin de vérifier si ce sont des équivalences directes ou s'il existe des spécificités dues à la langue ou à la culture scientifique. Par ailleurs, l'analyse diachronique lui permet d'éviter les pertes de contact avec l'évolution de la terminologie et l'état des techniques: en effet, la langue de spécialité évolue en fonction d'avancées scientifiques, des références théoriques prises par l'auteur du texte source et de sa volonté de se démarquer par rapport à la littérature existant sur son sujet.

En économie de l'énergie, le terme firewood initialement traduit par «bois de feu » ou «bois de chauffe », c'est-à-dire le bois utilisé comme moyen de produire de la chaleur, a aujourd'hui pour équivalent le terme "bois-énergie » dans les documents d'organismes spécialisés tels qu'EDF.

De même, diverses traductions ont été proposées pour le terme regulator, telles que " commission de surveillance » ou "organisme de contrôle ", avant que ne s'imposent « régulateur, autorité de régulation, instances de régulation », la sélection étant effectuée par les spécialistes du domaine.

Enfin, rappelons que la terminologie relative au développement a évolué dans le temps en illustrant progressivement l'emprise du procédé d'euphémisation. Ainsi, Third World countries et under-developed countries, comme leurs équivalents français «pays du Tiers Monde » et "pays sous-développés ", ont-ils fait place à less developed countries ou à developing countries, les « pays moins développés » ou " pays en (voie de) développement ». Toutefois, les PMA («pays les moins avancés»), dont l'équivalence en anglais est least developed countries, sont communément cités aujourd'hui en dépit d'une dénotation dépréciative dans les deux langues (Percebois $2002: 185$ ).

\subsection{Fidélité et circonstances de la traduction}

La notion de fidélité a suscité des prises de position diverses parmi les traducteurs, non pas tant quant à la nécessité d'être fidèle qu'au sens que l'on accorde au terme de " fidélité ».

En 1993, Jean Delisle définissait les critères majeurs de la fidélité comme la 
qualité d'une traduction qui respecte le plus possible le sens présumé du TD (exactitude quant au fond) et dont la formulation en LA est conforme aux usages et à la stylistique de cette langue (qualité quant à la forme). Les critères de fidélité varient selon la stratégie de traduction adoptée (libre, littérale) et selon le domaine traité, les genres et la fonction des textes. (1993:32) dans l'expression « fidélité au sens dans la fonctionnalité »:

En fonction du but choisi, le traducteur emploie des méthodes différentes, qui aboutissent à des résultats différents («traduction-adaptation", "traductionérudition ", «traduction littérale ») et s'éloignent parfois du sens. C'est pourquoi je parle de fidélité au sens dans la fonctionnalité, mais en la définissant (comme les autres dimensions de la fidélité au sens) comme nécessaire et limitée : nécessaire, car on a vu que l'équivalence de sens est dynamique et contextuelle par nature; limitée, car la «traduction-adaptation », la «traduction-érudition », sortent du domaine du sens. (1990: 218)

La notion de fidélité est perçue par le traducteur en fonction des circonstances de la traduction,ce que les traductologues anglophones qualifient de translation situation. Pourquoi traduit-on des textes anglais en français en contexte économique? Cette question vise à rechercher des raisons en amont et des objectifs en aval ; ce faisant, elle nous amène à nous interroger sur l'influence du commanditaire de la traduction mais aussi sur celle du ou des destinataire(s) de la traduction.

En amont de la traduction se trouve une rupture dans le continuum de la communication (Percebois 1999 : 31). L. Hewson et J. Martin précisent :

Translation does not just "happen», but results from (1) a need, and (2) an order. The need quite simply corresponds to a foreseen or actual breakdown in communication; the order corresponds to the instructions given by the TI to ensure that communication takes place. $(1991: 113)$

Le traducteur peut en effet dépendre d'un commanditaire externe de la traduction, celui que L. Hewson et J. Martin appellent Translation Initiator (TI), ce dernier étant ou non l'auteur du texte-source. Aussi, soulignerons-nous l'importance de l'identité socioculturelle de ce (ou de ces) commanditaire(s) de la traduction. 

ment à traduire : c'est le décodage du texte-source, qui permet au traducteur de reconnaître le domaine et les sous-domaines abordés, les registres de langue présents, les différents types de phrase, les figures de style, et de mettre en évidence les difficultés spécifiques de traduction (Delisle 1993). D’ailleurs, nous avons trouvé le terme de « code » dans la citation de M. Pergnier (voir 1.1.).

31 Cette étape sollicite la mobilisation de connaissances thématiques connexes pour effectuer la reconstruction du sens du texte à traduire, ces connaissances du domaine disciplinaire étant essentielles pour garantir la crédibilité de la traduction. Ainsi se forme une image mentale que le traducteur doit exprimer dans la langue-cible; il doit « retrouver le vouloir dire de l'auteur » (Seleskovitch $2001: 22$ ). 


\subsection{L'identification du contexte dans le domaine économique de référence}

32 À ce stade, nous préférons « identification du contexte " plutôt que « contextualisation » car ce dernier terme peut aussi bien correspondre à l'identification du contexte qu'à l'adaptation au contexte du texte d'arrivée.

Le traducteur doit identifier le contexte précisément. Un terme / une unité de traduction peut figurer dans différents contextes et se traduire à chaque fois de façon différente ou bien de la même façon dans certains contextes et différemment dans un ou plusieurs cas particuliers. Ce qui est vrai de termes employés dans un contexte non spécialisé - la valeur de background diffère dans background paper, background reading, background information (de base, de fond), academic background (formation universitaire) - semble encore plus évident en langue de spécialité.

Par exemple, l'emploi du terme diversion dans l'expression trade diversion lui fait prendre la valeur d'un concept, celui de "détournement d'échanges"; nous sommes dans le contexte du commerce international. Il en est de même pour le terme extension dans agricultural extension, qui prend le sens de "vulgarisation des nouvelles technologies agricoles ", extension ayant là un emploi très spécifique puisque les extension agents sont des intermédiaires qui vont, par exemple, transmettre de nouvelles techniques de production agricole; cette expression s'emploie dans le contexte de l'aide internationale aux pays en développement.

Observons les traductions du terme anglais alternative, substantif ou adjectif. Dans le contexte de l'économie de l'énergie, nous rencontrons energy alternatives pour rendre l'expression française "énergies de substitution", tandis que, dans le contexte de l'économie de projet, project economics, on étudie des project alternatives, des « variantes de projet ».

On peut ainsi multiplier les exemples: dans le domaine agricole ou l'aménagement du territoire, to reclaim land signifie "défricher », mais aussi « irriguer » lorsque l'action se passe dans un désert ou encore " assécher » lorsqu'elle se situe dans un marais.

Les acceptionsd'amenities peuvent se classer en deux groupes: d'une part, ce terme signifie " agréments, attraits », d'autre part, il a le sens d'« infrastructures, installations ». Les traductions de ce terme varieront en fonction des emplois, éventuellement dans un même contexte comme celui de l'économie de l'environnement; loss of amenities peut se traduire alors par "pertes d'agréments ", qui implique, par exemple, la moindre valeur touristique des abords d'un lac due à la pollution du plan d'eau, alors que dans les expressions tourist amenities ou rural amenities, amenities se traduira par «infrastructures ».

\subsection{Documentation}

La deuxième étape est celle de la documentation. Sur le plan pratique, le traducteur établit progressivement une liste de domaines de documentation indispensables. Cette documentation nécessite la collecte et l'investigation d'un corpus comparable dans les deux langues. Ce corpus réunira de préférence des documents contemporains du texte source et de même niveau scientifique. 
39 Le traducteur doit s'entourer d'un éventail d'aides à la traduction, sources écrites (dictionnaires, en particulier d'antonymes et de synonymes, encyclopédies), électroniques (banques de données ou dictionnaires en ligne ${ }^{1}$; sites Internet réunissant des liens spécialisés en économie ${ }^{2}$ ), ou orales, par interrogation de spécialistes du domaine. Sont également primordiales la recherche et la consultation de références précises au domaine dont relève le texte-source, telles que manuels ou articles de recherche, références adaptées au degré de scientificité de ce texte. L'insuffisance des connaissances du traducteur peut entraîner des imprécisions ou maladresses et donc une perte de crédibilité du texte d'arrivée nuisant fatalement à l'auteur du texte-source.

\subsection{La conversion du texte-source}

Troisième étape, la conversion du texte-source est parfois qualifiée de « transcodage ». Ce dernier terme convient parfaitement lorsqu'il est employé dans le sens, défini par Jean Delisle, d'une « opération consistant à établir des correspondances entre deux langues, soit au niveau du lexique soit au niveau de la phrase isolée » (1993:48), mais non dans celui que fustigent Claude et Jean Demanuelli (1991: 241) dans la définition suivante : "Aussi connu sous le nom de traduction automatique considérée comme 'anémique'; recouvre à la fois le calque et la traduction littérale ». En langue de spécialité, bien évidemment, ce dernier transcodage serait tout à fait insuffisant.

Le traducteur procède au découpage: c'est la délimitation des unités de traduction, l'unité de traduction (UT) étant, sur le plan linguistique, le «segment d'un énoncé dont les éléments lexicaux concourent à l'expression d'un seul élément de sens » (Delisle 1993 : 49). Dans son étude détaillée de l'unité de traduction (2003: 73-76), Michel Ballard précise : «c'est par rapport à la reformulation, autour de l'équivalence, que se construit l'UT ». En effet, le traducteur doit veiller à donner un caractère idiomatique et authentique au texte traduit.

Nombreux sont les risques d'erreurs dues à un mauvais découpage et à une extrapolation du sens, à l'égard desquels, en didactique de la traduction, il faut mettre en garde les traducteurs débutants. Nous citerons comme exemple d'erreur, due, en l'occurrence, à la polysémie de resort, la traduction par un étudiant de " the collapse of the talks in the Mexican resort of Cancun" par "l'échec du recours mexicain à Cancun", et celle de "the Doha Round, as the talks are known " par "d'après ce que nous savons des négociations, le cycle de Doha... ». Nous reviendrons ultérieurement sur la question de la polysémie.

Pour mettre en évidence les UT, le traducteur procède à une explication de texte, c'est-àdire une analyse précise du TD qui vise à dégager la signification précise des termes et des collocations. «Elle porte aussi sur les effets stylistiques, les sous-entendus, les allusions, les registres de discours, le rythme, la tonalité » (Delisle 1993 : 30).

Le texte à traduire présente un discours susceptible d'inclure diverses composantes qui s'interpénètrent. L. Hewson et J. Martin emploient le terme de «discourse family ", chaque «famille» de discours étant composée de «sous-familles». En outre, ils observent que ces familles ne sont pas séparées les unes des autres de façon hermétique, « as literary discourse permeates advertising and journalistic discourse (and vice versa) » (1991 : 118). Ainsi le discours économique intègre-t-il fréquemment des éléments du discours juridique.

Chaque texte-source peut exposer des cas d'interpénétration de différents discours en fonction de son sujet spécifique, par exemple le discours de l'économie en général, de 
l'économie de l'énergie en général (c'est-à-dire commun à tous les domaines), de l'économie des hydrocarbures, de l'économie de la gestion des risques (lié à l'emploi des hydrocarbures: transport, transformation). Il importe de les reconnaître pour leur attribuer, dans la traduction, les spécificités de leur terminologie respective en tenant compte de leurs champs notionnels.

Devant une difficulté, le traducteur étudie les possibilités de reconstruction du textesource pour mettre en balance diverses options de traduction en langue et culture d'arrivée. Cette reconstruction se fait à partir de paraphrases, la paraphrase étant le développement explicatif d'un texte. Ainsi le texte d'arrivée résulte-t-il de la comparaison entre les différents énoncés paraphrastiques du texte-source et de leurs équivalences dans la langue d'arrivée. Si le traducteur est en relation avec l'auteur du texte, la communication qui s'établit entre eux peut même être l'occasion de modifications du texte source, afin de le clarifier ou de le préciser (Percebois 1999 : 39-40).

En définitive, l'art du traducteur dépend de sa maîtrise de la terminologie et des techniques de rédaction, de sa connaissance des conventions propres à chaque type de textes (article de recherche, document de vulgarisation) et de son aptitude à répondre aux attentes aussi bien de l'auteur que des destinataires de sa traduction.

\section{3. Équivalences attestées et détours des choix traductologiques}

\subsection{L'équivalence}

L'équivalence consiste à rendre une expression figée de la langue de départ par une autre dans la langue d'arrivée. Michel Ballard propose une «typologie des schémas d'équivalence» très complète (2003: 77-86), qu'il partage en deux catégories: l'équivalence directe « où l'on a un effet de simple substitution entre des termes et des structures qui semblent se correspondre » et l'équivalence indirecte « où l'aboutissement offre des différences de diverses natures (autres que la graphie) par rapport à la base ».

Nos équivalences, "attestées" par une étude comparative des composants anglais et français de notre corpus, se présentent également en diverses catégories. Elles vont ainsi de l'équivalence directe totale, ou expression figée, consacrée par l'usage, à l'équivalence indirecte, beaucoup plus fréquente, avec, par exemple, des modifications morphosyntaxiques ou une différence de concentration.

Dans les premiers temps d'une recherche terminologique, le traducteur doit découvrir les équivalences à partir de ses propres références dans les deux langues. Il va ainsi découvrir des idiotismes, l'idiotisme étant une « expression propre à une langue et qui n'est pas traduisible littéralement dans une autre langue » (Delisle 1993: 33). Traduisant le plus souvent vers sa langue maternelle, le traducteur n'aura d'ordinaire pas de difficulté à rendre les idiotismes; ceci n'est pas vrai de la traduction de documents en langue de spécialité lorsque le traducteur n'est pas lui-même un spécialiste du domaine de référence.

51 Comme exemples d'expressions figées en anglais et en français de spécialité économique, nous citerons :

tax basis : l'assiette fiscale

break-even point : le seuil de rentabilité 
infant industries : les industries naissantes

government procurement ; les marchés publics

workable competition : la concurrence effective (Percebois 1982 : 213)

rainfed crops; les cultures sèches; cultures non irriguées (explicitation), cultures

alimentées par les eaux pluviales (périphrase) d'emploi de termes associés de façon typique. À cet égard, André Clas évoque «la combinabilité, c'est-à-dire l'aptitude qu'ont les mots à se joindre à d'autres mots » (1994 : 576). De ce fait, à l'étape de la conversion, les collocations jouent un rôle essentiel dans la composition du texte d'arrivée. Selon la définition de Michel Ballard

Une collocation est un syntagme figé, dont les termes entretiennent des relations de compatibilité sémantique privilégiées et dont l'agencement sera reconnu comme naturel ou usité par un locuteur natif ou compétent. (2003 : 191) l'ambiguïté et identifier l'emploi spécifique des polysèmes car un énoncé peut faire l'objet de plusieurs interprétations linguistiques. Citons l'exemple de breakdown qui signifie communément "effondrement, rupture, échec ", mais prend des sens spécifiques dans operations breakdown: la "décomposition des tâches" ou occupational breakdown of the population : la « répartition de la population par emplois ». est l'abréviation de purchasing power parity,la " parité des pouvoirs d'achat» mais également de polluter pays principle, le "principe du pollueur-payeur", tandis que LSE peut signifier London School of Economics aussi bien que London Stock Exchange.

Termes de deux langues différentes « dont les signifiants sont en relation d'homonymie ou de paronymie et dont les signifiés diffèrent plus ou moins » (Ballard $2003: 260$ ), les faux amis abondent en langue de spécialité et peuvent être source de confusion. Ce sont parfois des faux amis complets - tels que commodities dont le sens générique est « biens et services ", mais dont les acceptions sont multiples - souvent des faux amis partiels. Ainsi, 
par exemple, une analyse synchronique de l'anglais et du français montre que des différences culturelles ont donné un sens particulier à certains homographes. En anglais, on entend par legislature le corps législatif ou l'assemblée législative, c'est-à-dire un groupe de personnes ayant le pouvoir de promulguer et de modifier les lois. En français, si le terme « législature " peut avoir ce sens, que le Petit Robert signale comme " rare ", il représente habituellement la «durée du mandat d'une assemblée législative " (Petit Larousse), signifié qui se rendrait habituellement en anglais par term of office.

\subsection{Détours des choix traductologiques}

Ces « détours » sont envisagés et testés par le traducteur dans le cas des équivalences indirectes. Ils apparaissent dès que la créativité du traducteur est mise à contribution, lorsqu'il ne se trouve pas en présence d'équivalences figées. En langue de spécialité, ici en anglais et en français économiques, le traducteur doit particulièrement faire appel à des connaissances techniques du domaine d'application pour que sa créativité ne l'éloigne pas de la précision scientifique.

Les options périphrastiques envisagées par le traducteur sont des hypothèses de travail. Elles offrent diverses recompositions du texte d'arrivée parmi lesquelles s'effectueront ses choix traductologiques. Ces choix s'imposent plus ou moins directement après observation de l'environnement de l'unité de traduction considérée.

Certaines difficultés sont dues à l'existence de termes qui se suffisent en anglais et nécessitent un étoffementen français. L'étoffement consiste à employer plus de mots en français qu'en anglais pour exprimer la même idée. Citons, par exemple :

Dumped goods : produits vendus au-dessous de leur prix de revient

Second-best theory : théorie de l'optimum de second rang

Dans ces deux cas, il est vrai, le maintien du terme anglais, dumping, ou second best, dans les équivalences françaises, permettrait évidemment de les raccourcir, ce qui donnerait " prix de dumping », « théorie du second best ».

Le terme policy présente des difficultés de traduction récurrentes en contexte économique, difficultés qui trouveront diverses solutions, telles que l'adjonction d'un adjectif en français après identification du contexte précis; ainsi, en fonction de son insertion dans la phrase, policy decision pourra se traduire par «décision de politique économique / environnementale / financière", etc., mais aussi par "décision stratégique ( (entreprises, management) ou encore «décision de principe» (administration publique), tandis que policy instrument pourra se rendre par « instrument d'intervention » ou « instrument stratégique ».

Par ailleurs, la concision des compositions nominales et adjectivalesen anglais accroît la difficulté de leur conversion en français.

Pour les noms comme pour les adjectifs composés, la conversion en français donnera une équivalence dans le texte d'arrivée déjà amplifiée par les connecteurs souvent indispensables à la liaison des termes en français : commodity basket est équivalent au " panier de la ménagère » tandis que price-elastic demand est une « demande élastique par rapport au prix ». Ce sont là deux exemples très simples. Mais d'autres cas se révèlent d'une complexité source de nombre de « détours » envisagés et parfois conservés dans la traduction. 
67 Si la traduction de decision maker ne présente pas de difficulté,il n'en est pas de même de policy maker : ce dernier pourra être « responsable » ou « concepteur » d'une politique ( energy policy maker, environmental policy maker, economic policy maker), un "stratège ", "théoricien", "technocrate", "dirigeant» dans une institution, ou un "décideur politique ». C'est l'art du traducteur qui lui fera trouver dans la langue d'arrivée le terme en parfaite adéquation avec le sens du texte-source.

La difficulté augmente encore lorsque le nom composé considéré est intégré dans une théorie. Dans la théorie du monopole, on observe le pouvoir de négociation de celui qui fixe le prix : «the price maker's bargaining power » (Percebois 1982 : 159). Dans la théorie de l'oligopole, le price leader est la firme dominante dont dépend le prix fixé, les autres étant des price followers (Percebois 1982: 195) qui appliquent promptement les variations de prix impulsées par le price leader. Il n'existe pas de traduction «figée» de ces noms composés. Toutefois, dans un manuel de théorie économique français, nous avons trouvé l'expression « firmes 'preneuses de prix' », vrai « calque » faisant explicitement référence à l'emploi de price taker (Percebois $2002: 56$ ).

69 La traduction des adjectifs composés peut également donner lieu à la mise en balance de diverses périphrases. Labour-intensive et capital-intensive offrent des exemples pour lesquels la traduction varie sensiblement en fonction du public cible. Pour un public n'entendant pas le discours économique spécialisé, a labour-intensive production process ou capital-intensive production process sont des processus de production « exigeant beaucoup de main-d'œuvre ou beaucoup de capital», ou, traduction plus technique, "à forte intensité de main-d'œuvre » ou «à forte intensité de capital », tandis que les spécialistes parlent de «processus de production non-capitalistique » ou «processus de production capitalistique ", termes prêtant à confusion pour l'auditeur non averti. En effet, pour employer à bon escient ces dernières dénominations, il faut également avoir assimilé le sens de l'adjectif «capitalistique» qui fait partie de la terminologie économique technique : il qualifie des biens qui exigent une plus forte proportion de capital que de travail pour être produits.

70 Sur ces modèles ont été formés d'autres adjectifs composés incluant l'adjectif intensive tels que : information-intensive, energy-intensive, R\&D-intensive, knowledge-intensive, researchintensive. Chacun de ces adjectifs pose des difficultés spécifiques de traduction. Citons, par exemple, l'expression trade in primary and natural resource-intensive commodities faisant référence au commerce de biens dont la production exige une forte proportion de produits de base et de ressources naturelles (Percebois $2002: 62$ ), ou l'emploi de pollutionintensive dans la question suivante :

Will freer trade lead to concentrations of pollution-intensive industries in developing countries - turning them into "pollution-havens"?

La libéralisation du commerce entrainera-t-elle une concentration des industries fortement polluantes dans les pays en développement, les transformant ainsi en « havres de pollution » ou «paradis pour pollueurs»?

71 Pollution-intensive est utilisé de façon typique pour qualifier les industries, mais on trouvera aussi pollution-intensive firms/trade/products/production/ goods/commodities. Notons au passage la construction de pollution-haven sur le modèle de tax-haven: " paradis fiscal », les « détours » des deux propositions de traduction se justifiant par le problème particulier posé par les deux éléments antonymiques de ce nom composé.

72 Freer trade - que nous traduisons par « libéralisation du commerce »- est un exemple de faux comparatif, «adjectif qualificatif employé au degré comparatif dans une 
comparaison dont le second terme n'est pas précisé » (Delisle 1993 : 31). Par un procédé de modulation, cette construction elliptique, très fréquente en anglais, se rendra souvent par un simple nom ou un simple adjectif en français : major nuclear powers traduit par « les grandes puissances nucléaires » en est un exemple typique. Nous en trouvons trois autres dans la phrase ci-dessous :

This step would be combined with stricter regulations and better enforcement of such tighter controls.

Cette étape s'accompagnerait d'un durcissement de la réglementation et d'une meilleure application de contrôles renforcés de ce type.

Cette étape serait assortie d'un renforcement de la réglementation doublé d'une application moins laxiste de contrôles rigoureux de ce type.

La traduction de better enforcement par « application moins laxiste » illustre le procédé de négation du contraire. La négativation fréquente du discours français donne ainsi lieu à l'évocation de « détours » envisageables dans la traduction.

Autre source de "détours » dans la reformulation, certaines expressions typiques du discours du chercheur dont voici un exemple :

We find strong relationships between MWTP and individual characteristics, such as age, income and education, but little evidence that MWTP varies systematically with economic growth.

Nous constatons une forte corrélation entre le consentement marginal à payer des individus et les caractéristiques de chaque individu, telles que son âge, son revenu, son éducation, mais peu de preuves d'une variation systématique du CMP avec la croissance économique.

Nous observons [...] mais il existe peu de preuves démontrant que ...

Selon nos observations, il existe une forte corrélation [...] mais peu de signes tendant à prouver que le CMP varie systématiquement en fonction de la croissance économique.

Les phrases longues sont typiques de l'article de recherche en économie. Si la fidélité veut la traduction à l'identique, un choix traductif consiste à segmenter ces phrases en articulant, c'est-à-dire en introduisant des charnières telles que "en outre ", "par conséquent », «ensuite», «ainsi », « mais »; J. Delisle les définit comme un « mot ou groupe de mots servant à lier les parties d'un discours de manière à faire ressortir les rapports logiques les unissant» (1993: 22). Si la segmentation efface la relation interpropositionnelle et lui substitue un point, ce qui crée deux phrases plus simples à partir d'une phrase complexe, on obtient l'effet inverse par la désegmentation de deux phrases courtes telles que :

In Europe, the $92 \mathrm{GW}$ was installed in 1995, generating a total of $307 \mathrm{TWh}$. This accounted for $92 \%$ of the renewable energy generated.

En Europe, $92 \mathrm{GW}$ ont été installés en 1995, produisant un total de 307 TWh, ce qui représente $92 \%$ de l'énergie renouvelable produite.

Une fois que le traducteur a décelé dans le texte à traduire la présence de termes ou d'expressions pour lesquels ses recherches, ou celles de ses prédécesseurs, ont mis en évidence des équivalences "attestées", il peut laisser libre cours à sa créativité pour rendre compte de formulations plus ou moins complexes. Nous avons voulu citer cidessus certaines de ces difficultés et donner quelques exemples des possibilités de choix traductologiques qui se présentent à l'esprit du traducteur dans le passage de l'anglais au français en contexte économique. 


\section{Conclusion}

Traduire, c'est reformuler un texte dans une autre langue en étant fidèle à son sens et en concevant une forme adaptée à la langue et à la culture du public auquel cette traduction est destinée, en l'occurrence la communauté linguistique des économistes aussi bien que celle des autres usagers de la langue économique, à un moindre degré de scientificité.

Pour cela, le traducteur se livre à des arbitrages; il doit préserver le sens du texte de départ tout en tenant compte des spécificités linguistiques et culturelles des destinataires dans le texte d'arrivée. De ce fait, les critères majeurs de ses choix traductifs seront l'objectif de clarté, c'est-à-dire la facilité d'identification du concept de référence pour le public cible; l'objectif de fidélité à l'original, au cadre technique de l'information communiquée, au propos et à l'esprit du document original; enfin la nécessité de préserver la cohérence synchronique des éléments constitutifs du texte d'arrivée et de respecter l'évolution diachronique du lexique dans le choix des termes.

Dans la traduction de documents économiques d'anglais en français, la principale source de difficulté n'est pas liée à la technicité des termes ou des expressions. Elle tient essentiellement à la mise évidence de leur acception contextuelle dans le texte soumis à la traduction et à ce que Pierre Lerat appelle «la recherche de l'adéquation communicative » $(1995: 102)$.

\section{BIBLIOGRAPHIE}

Ballard, Michel. 2003. Versus : la version réfléchie, repérages et paramètres. Paris : Ophrys.

Cabré, Maria Teresa. 1998. La terminologie : théorie, méthode et applications. Ottawa: Les Presses de l'Université d'Ottawa \& Paris : Armand Colin.

Clas, André. 1994. «Collocations et langues de spécialité ». Meta 34/4, 576-580.

Delisle, Jean. 1993. La traduction raisonnée. Ottawa : Presses de l'Université d'Ottawa.

Delisle, Jean. 2001. « L'évaluation des traductions par l'historien ». Meta 46/2, 209-226.

Demanuelli, Claude \& Jean Demanuelli. 1991. Lire et traduire, anglais-français. Paris : Masson.

Demanuelli, Claude \& Jean Demanuelli. 1995. La traduction : mode d'emploi. Paris : Masson.

Gouadec, Daniel. 1990. Le traducteur, la traduction et l'entreprise. Paris : AFNOR.

Hewson, Lance \& Jacky Martin. 1991. Redefining translation. The Variational Approach. Londres : Routledge.

Humbley, John. 1987. « L'emprunt sémantique dans la terminologie de l'informatique ». Meta $32 / 3,321-325$.

Hurtado, Albir Amparo. 1990. La notion de fidélité en traduction. Paris : Didier Érudition.

Ladmiral, Jean-René. 1994. Traduire : théorèmes pour la traduction. Paris : Gallimard. 
Lerat, Pierre. 1995. Les langues spécialisées. Paris : Presses Universitaires de France.

Percebois, Jacqueline. 1982. L'anglais de la microéconomie. Paris : Economica.

Percebois, Jacqueline. 1999. «L'anglais de l'économie : recherches en terminologie, lexicologie comparée de l'anglais et du français, traductologie et didactique ». Synthèse présentée à

l'Université Montpellier 1 pour l'obtention de l'Habilitation à Diriger des Recherches.

Percebois, Jacqueline. 2002. Terminologie Anglais-Français du Commerce International, Théories, Politiques, Accords et Institutions. Paris : Economica.

Pergnier, Maurice. 1993. Les fondements socio-linguistiques de la traduction. Lille : Presses Universitaires de Lille.

Seleskovitch, Danica \& Marianne Lederer. 2001. Interpréter pour traduire. Paris : Didier Érudition.

\section{NOTES}

1. Cf. Grand Dictionnaire Terminologique, Eurodicautom ou le Trésor de la Langue Française Informatisé.

2. Par exemple <http://liensutiles.org/econom.htm>.

\section{RÉSUMÉS}

Cet article traite de la traduction en français de textes anglais de spécialité économique. Il étudie d'abord les circonstances de la traduction et décrit les principales étapes de ce processus impliquant deux langues et deux cultures. En s'appuyant sur des principes théoriques de traductologie, il expose les traits caractéristiques de la traduction. La considération d'un corpus équivalent dans les deux langues révèle l'existence d'éléments terminologiques figés et met en évidence les diverses options périphrastiques entre lesquelles s'effectuent les choix traductologiques.

This article deals with the translation into French of English texts in the field of economics. First it studies the translation situation and describes the main steps of a process involving two languages and two cultures. Using theoretical traductology principles, it presents the main features of translation. The comparison of an equivalent corpus in both languages reveals the existence of stable terminology items and various periphrastic options among which translation choices can be selected.

\section{INDEX}

Mots-clés : anglais de spécialité, anglais économique, métalangage de la traduction, traduction, traductologie

Keywords : English for economics, English for Specific Purposes, traductology, translation, translation metalanguage 


\section{AUTEUR}

JACQUELINE PERCEBOIS

Jacqueline Percebois est professeur à l'université Aix-Marseille 1 (DEMA) où elle est responsable du Master Métiers de la traduction. Ses recherches, portant sur la terminologie comparée anglais-français en économie, ont donné lieu à la publication d'articles et de trois ouvrages sur les thèmes de la microéconomie, de la macroéconomie et du commerce international. Elle est membre de l'EA2025. Jacqueline.Percebois@up.univ-aix.fr 